\title{
Wave Function of the Proton in a Uniform Magnetic Field
}

\section{Dale S. Roberts}

Special Research Centre for the Subatomic Structure of Matter and Department of Physics, University of Adelaide 5005, Australia.

E-mail: dale.roberts@adelaide.edu.au

\section{Patrick O. Bowman}

Centre for Theoretical Chemistry and Physics and Institute of Natural Sciences, Massey University (Albany), Private Bag 102904, North Shore City 0745, New Zealand.

E-mail: P.O.Bowman@massey.ac.nz

\section{Waseem Kamleh*}

Special Research Centre for the Subatomic Structure of Matter and Department of Physics, University of Adelaide 5005, Australia.

E-mail: waseem.kamleh@adelaide.edu.au

\section{Derek B. Leinweber}

Special Research Centre for the Subatomic Structure of Matter and Department of Physics, University of Adelaide 5005, Australia.

E-mail: dleinweb@physics.adelaide.edu.au

\begin{abstract}
We calculate the wave functions of the $d$-quark in the ground state of the proton, and how it is affected in the presence of a uniform background magnetic field. We focus on wave functions in the Landau and Coulomb gauges. We observe the formation of a scalar $u$ - $d$ diquark pair within the proton in Landau gauge, which is not present in Coulomb gauge. The overall distortion of the wave functions under a very large magnetic field, as demanded by the quantisation conditions on the field, is quite small.
\end{abstract}

The XXVIII International Symposium on Lattice Field Theory, Lattice2010

June 14-19, 2010

Villasimius, Italy

\footnotetext{
*Speaker.
} 


\section{Introduction}

The wave functions of a baryon on the lattice provides an insight into the shape and properties of the particle. Further to this, the wave functions also provide a diagnostic tool for the lattice, being able to determine how well a particular state fits on the lattice volume. The earliest work on wave functions on the lattice was carried out on small lattices, for the pion and rho, initially in $S U$ (2) [1]. Further progress was made in the early nineties, where gauge invariant Bethe-Salpeter amplitudes were constructed for the pion and rho [2] by choosing a path ordered set of links between the quarks. This was then used to qualitatively show Lorentz contraction in a moving pion. Hecht and DeGrand [3] conducted an investigation on the wave functions of the pion, rho, nucleon and Delta using a gauge dependent form of the Bethe-Salpeter amplitude, primarily focusing on the Coulomb gauge.

The background field method [4] for placing an external electromagnetic field on the lattice has been used extensively in lattice QCD to determine magnetic moments of hadrons. Early studies on very small lattices with only a few configurations $[5,6]$ showed remarkable agreement with the experimental value of the magnetic moments of the proton and neutron. More recent studies on magnetic moments [7] show good agreement with experimental values of the magnetic moments of the baryon octet and decuplet. This method has also been extended to the calculation of magnetic and electric polarisabilities $[8,9]$. We are able to use the wave function to determine the effect of the background magnetic fields on the shape of the proton.

As background field methods have become more widely used, it is apparent that large uniform fields demanded by the quantisation conditions should cause some concern with regards to the calculation of moments and polarisabilities. It is entirely possible that the distortion caused by these fields could be so dramatic that the particle under investigation bears little resemblance to its zero-field form. For this reason, we will use the wave function as a tool to investigate the deformation caused by a background field on a particle like the proton.

\section{Lattice Techniques}

In order to calculate the wave function on the lattice, we modify the standard interpolating fields used in the two-point Greens functions such that at the sink,

$$
\chi_{P}\left(\vec{x}, \vec{y}, \vec{d}_{1}, \vec{d}_{2}\right)=\varepsilon^{a b c}\left(u_{a}^{T}\left(\vec{x}+\vec{d}_{1}\right) C \gamma_{5} d_{b}(\vec{x}+\vec{y})\right) u_{c}\left(\vec{x}+\vec{d}_{2}\right),
$$

where $\vec{d}_{1}=\left(d_{1}, 0,0\right), \vec{d}_{2}=\left(d_{2}, 0,0\right)$. For separations of the $u$ quarks across even numbers of lattice sites, $d_{1}=-d_{2}$, and for odd separations, $d_{1}+1=-d_{2}$. The normalisation chosen for the density distributions is to scale the raw correlation function data such that the sum over $\vec{x}$ and $\vec{y}$ from Eq. (2.1) is 1. We consider eight values for the separation of the quarks in Eq (2.1), between 0 and 7 lattice spacings.

We use an ensemble of 200 quenched configurations with a lattice volume of $16^{3} \times 32$, generated using the Luscher-Weisz $\mathscr{O}\left(a^{2}\right)$ improved gauge action [10]. The $\mathscr{O}(a)$ improved FLIC fermion action [11] is used to generate the quark propagators. We use $\beta=4.53$, corresponding to a lattice spacing of $a=0.128 \mathrm{fm}$, determined by the Sommer parameter, $r_{0}=0.49 \mathrm{fm}$. We employ 
50 sweeps of gauge invariant Gaussian smearing to the fermion source. Two values for the hopping parameter are used, $\kappa=0.12885$ and 0.12990 , corresponding to pion masses of $0.697 \mathrm{GeV}$ and $0.532 \mathrm{GeV}$. The gauge fields generated are fixed to the Landau and Coulomb gauges using the conjugate gradient Fourier acceleration method for improved actions [12], to an accuracy of 1 part in $10^{12}$.

A background electromagnetic field is placed on the lattice in the form of a phase, $U_{\mu}(\mathrm{x}, \mathrm{y})$, that multiplies the $S U(3)$ links [4]. This phase for a constant magnetic field in the $z$ direction given by

$$
U_{y}(x, y)=e^{i a e B x} .
$$

Due to the periodic boundary conditions, we also require

$$
U_{x}(x, y)=e^{-i a e B N_{x} y},
$$

along the boundary $x=N_{x}$, with all other $U_{\mu}(x, y)=1$. Furthermore, a quantisation condition of $e B=e B_{z}=\frac{2 \pi}{N_{x} N_{y} a^{2}}$ is induced by the boundary conditions.

\section{Zero-Field Results}

We begin by looking at the wave function without a background field in the Landau gauge. The wave function constructed from Eq. (2.1) is not symmetric around the centre of mass of the proton. We find a peak is centered around the $u$ quark that resides in the scalar pairing with the $d$ quark in Eq. (2.1). This leads us to believe that the $u$ and $d$ quark tend to form a scalar pair within the proton. At this point, we choose to symmetrise the $u$ quarks around the centre of the lattice, changing our annihilation operator from Eq. (2.1) to

$$
\begin{aligned}
\chi_{P}\left(\vec{x}, \vec{y}, \vec{d}_{1}, \vec{d}_{2}\right) & =\varepsilon^{a b c}\left(u_{a}^{T}\left(\vec{x}+\vec{d}_{1}\right) C \gamma_{5} d_{b}(\vec{x}+\vec{y})\right) u_{c}\left(\vec{x}+\vec{d}_{2}\right) \\
& +\varepsilon^{a b c}\left(u_{a}^{T}\left(\vec{x}+\vec{d}_{2}\right) C \gamma_{5} d_{b}(\vec{x}+\vec{y})\right) u_{c}\left(\vec{x}+\vec{d}_{1}\right) .
\end{aligned}
$$

This choice is motivated by the fact that the interpolating field places one of the $u$ quarks permanently within the scalar pair, however, physically, this would not be the case, as the $u$ quarks within the proton should be indistinguishable.

Upon implementing this symmetrisation, we see no evidence that diquark clustering is occurring at small $u$-quark separations. Rather, the wave function broadens and flattens around the centre of mass of the system. However, when we move to a separation of five or more lattice units, or $0.640 \mathrm{fm}$, we see two distinct peaks form. At this stage, the $u$ quarks are separated further than was considered in [3].

In the Coulomb gauge, diquark clustering is present as evidenced in the unsymmetrised wave function, however, the support in the centralized region hides the diquark clustering upon symmetrisation. Fig. 1 illustrates results for $u$ quarks separated by 7 lattice units.

In both the Landau and Coulomb gauges, the mass dependence of the wave functions is almost negligible, as there are no significant differences in the shape of the wave function when the quark mass is changed, as was noted in [1].

We note that there are several reasons that we are able to see the diquark clustering in the Landau gauge where Ref. [3] did not. Our use of large smeared sources, the averaging over $\vec{x}$ 

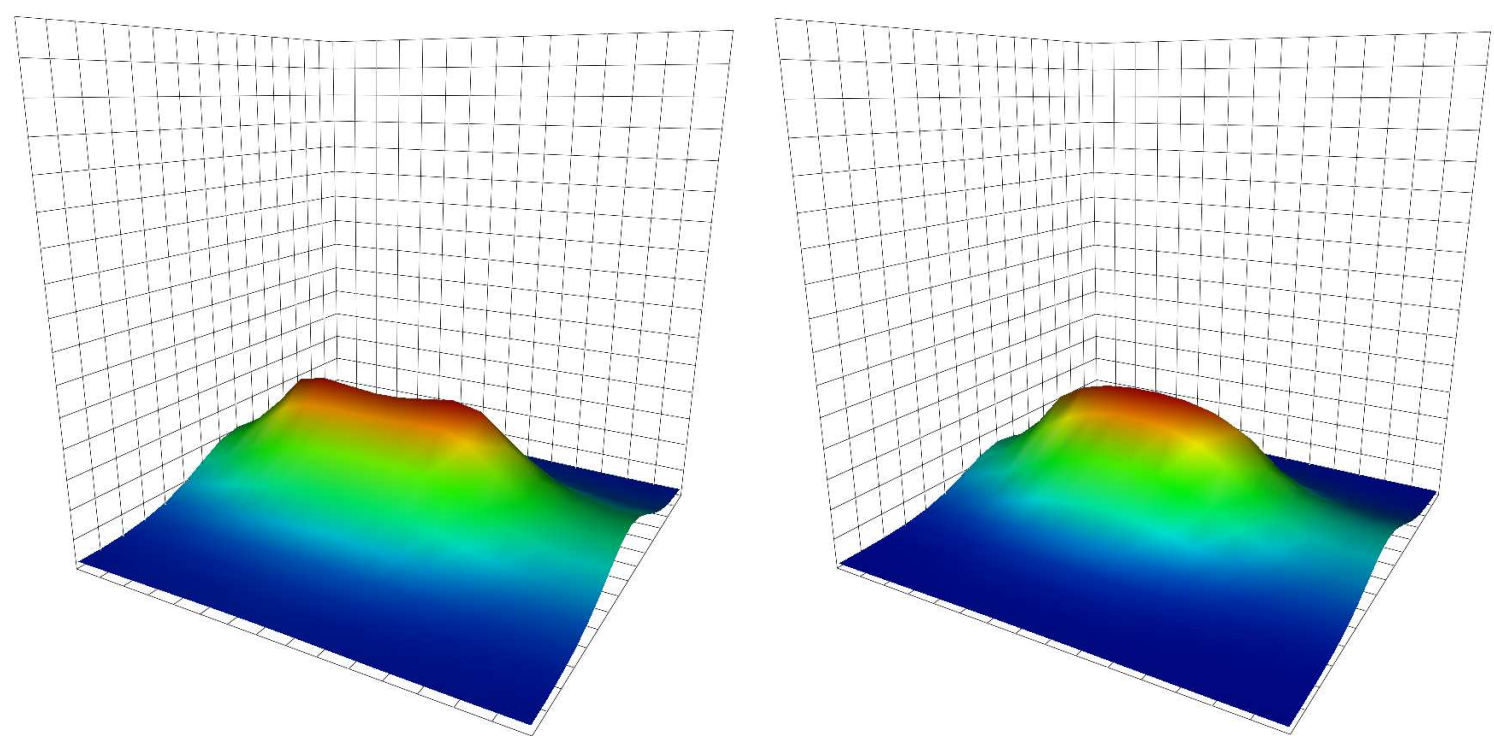

Figure 1: (Colour Online) The wave function for the $d$ quark in the plane of the $u$ quarks separated by 7 lattice units, in the Landau gauge (left), and the Coulomb gauge (right). Two distinct peaks have formed over the location of the $u$ quarks in the Landau gauge wave function, whereas a single, broad peak is visible over the centre of mass of the system in the Coulomb gauge. The scale is such that the largest value of all of the fixed quark separations will sit at the top of the grid, with all other points of the wave function scaled accordingly.

in Eq. (2.1), as well as using improved actions for both the quarks and the gauge fields and the consideration of hundreds of gauge fields provides far better statistics, allowing access to further $u$ quark separations with a high signal-to-noise ratio, as well as investigating lighter quark masses. Furthermore, our lattices extend twice as far in the temporal direction, thus reducing the chance of any contamination from states propagating through the periodic boundary conditions.

Although models featuring diquarks within hadrons have been used extensively for many years [13], there has been little, if any, direct evidence for the existence of such a cluster within a particle. Earlier lattice studies that have paired two light quarks with a static quark $[14,15]$ have shown a large diquark $(\mathscr{O}(1) \mathrm{fm})$ can form inside of a baryon, though with limited effect on the structure of the particle. More recently, light quarks have been paired with various diquark correlators [16] which suggest that diquarks are not a significant factor in light baryons. To the best of our knowledge, this is the first time that such a diquark configuration has been shown in a baryon composed of three light quarks.

\section{Background Magnetic Field Results}

The first notable result from the use of the aforementioned method of placing a background field on the lattice is that an asymmetry is produced in the direction of the vector potential. This asymmetry occurs in both the Landau gauge and Coulomb gauge to the same extent. This is an unphysical result of the method in which we place the field on the lattice, which can be shown by applying the gauge transformation $G(x, y)=e^{-i B x y}$ to the field described above. Upon doing this, the asymmetry in the wave function can be seen to move to the direction of the vector potential 
once again as shown in Fig. 2. In order to minimise the gauge effect of the choice of background field on the wave function, we choose an average over four implementations of the background field. The two implementations described above and two in which a gauge transformation has been applied such that the magnitude of the vector potential decreases across the lattice.
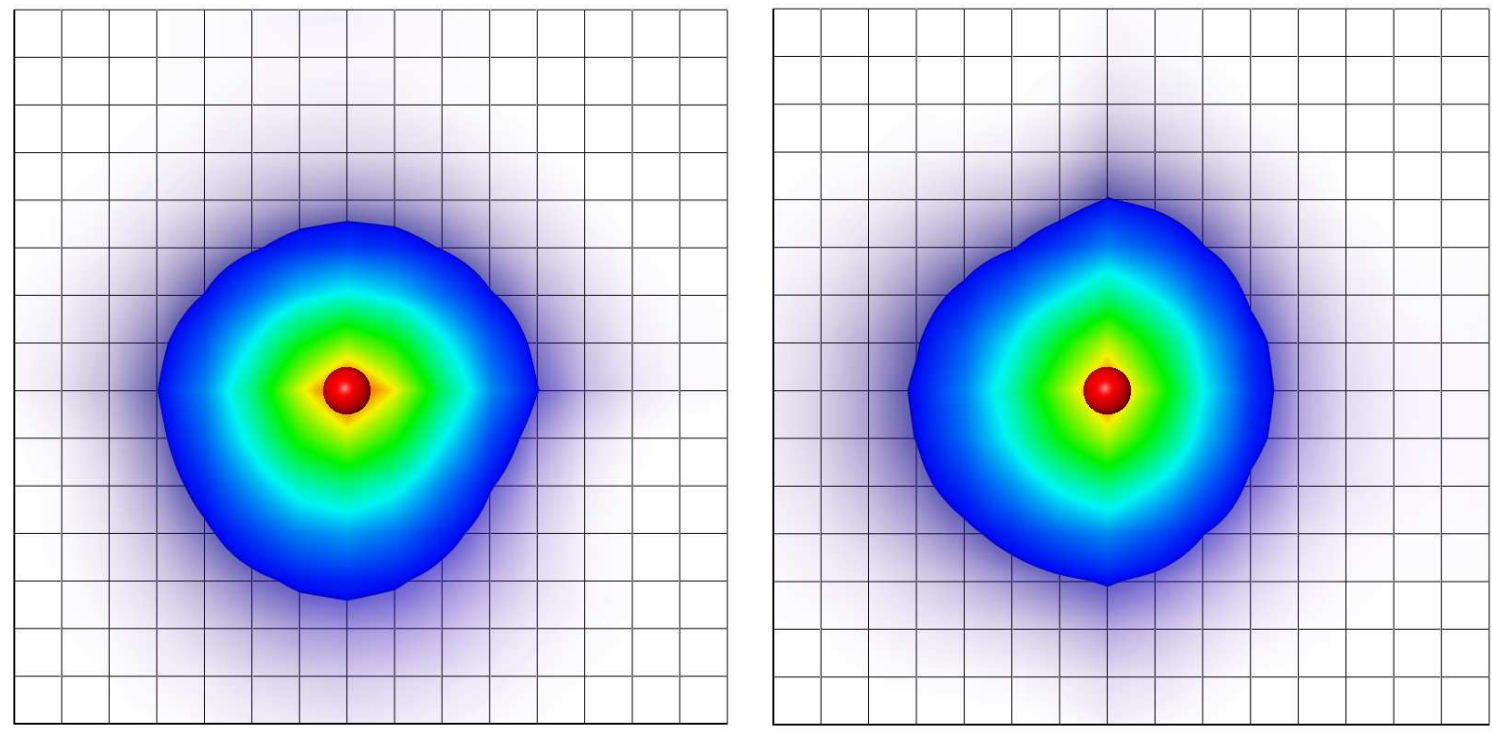

Figure 2: (Colour online) The wave function for the $d$ quark cut in the plane of the $u$ quarks, in the presence of a background magnetic field in the Landau gauge, with the original implementation (left), and upon implementing the gauge transformation described above (right). In this image, the field, $\vec{B}$ is pointing into the page. There is a clear asymmetry perpendicular to the field that changes with the vector potential, $A_{\mu}$, in spite of the background field not changing.

Once averaging over the four vector potentials has been applied, we look at the wave function in the $x z$-plane. In spite of the very large magnetic field strength imposed by the boundary conditions, the change in the wave function is quite small, with approximately one half lattice spacing deviation from being spherical (Fig. 3). This subtle result is consistent with that expected from the polarizability as the current experimental value for the proton polarizability is $\beta_{M}=1.9(5) \times 10^{-4} \mathrm{fm}^{3}$ which gives the second order response to the field of around, $\frac{1}{2} \beta_{M} e^{2} B^{2}=0.4 \mathrm{MeV}$. Compared to the zero field results, the wave function is enhanced closer to the centre, and falls off faster.

As the quarks are separated, the wave functions in the background field tend to be more localized than the same wave functions without a background field. Some stretching along the field orientation at the centre of the distribution is apparent, making the distribution more spherical (Fig. 4).

\section{Conclusion}

In this study, we have performed the first examination of the wave function of the proton in the presence of a background magnetic field in both the Landau and Coulomb gauges. We have shown that there is a distinct difference between the wave functions in the Landau and Coulomb gauge, with the Landau gauge exhibiting clear diquark clustering, whereas the wave functions in 

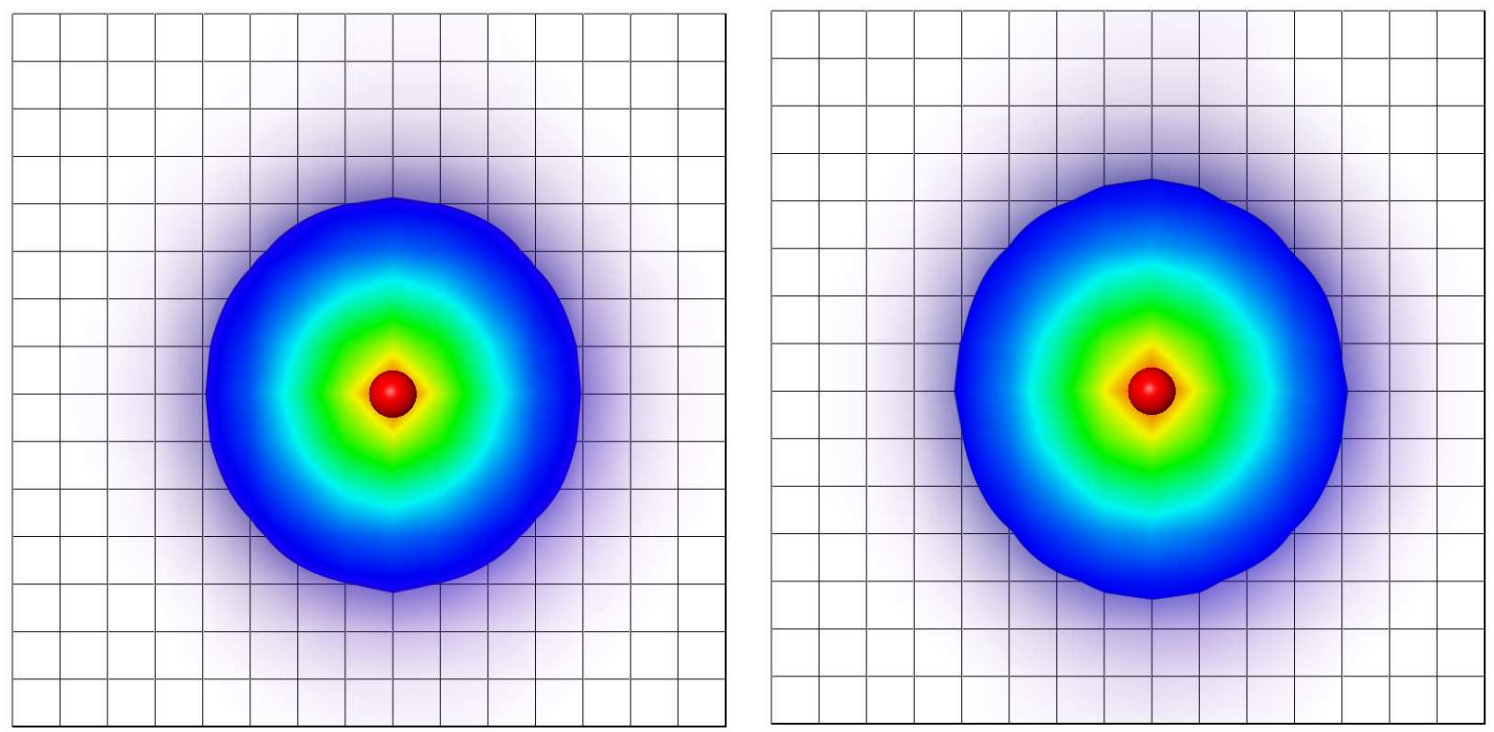

Figure 3: (Colour online) The wave function from for the $d$ quark cut in the plane of the $u$ quarks, after symmetrising the vector potential, $A_{\mu}$ in the presence of the background magnetic field, $B$, in the Coulomb gauge (left), and in the Landau gauge (right). In this image, the field is pointing to the top of the page. In spite of the magnitude of the field, a fairly small deviation from spherical is seen in both gauges, although slightly more pronounced in the Landau gauge.
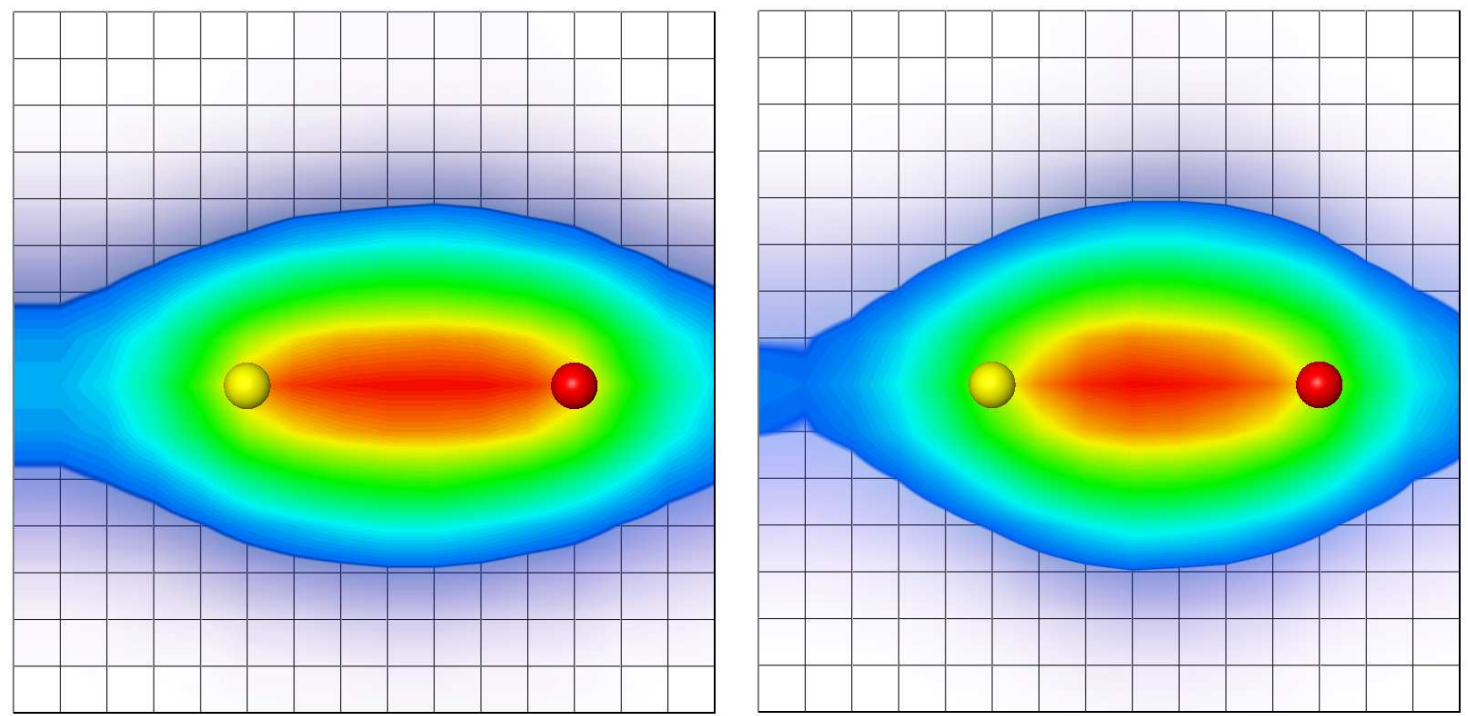

Figure 4: (Colour online) The wave function in the Coulomb gauge cut in the $x-z$ plane of the $u$ quarks, which are separated by seven lattice units in the transverse direction with zero background field (left) and in the presence of the field (right). The direction of the field is up the page and the spheres denote the positions of the $u$ quarks. The smallest value shown for the wave function is $20 \%$ of the peak value. 
the Coulomb gauge did not. The wave functions in the Landau gauge were broader than those in the Coulomb gauge. We have also confirmed that the wave function exhibits very little, if any, mass dependence.

On the application of the background field, we revealed a gauge-dependent change in the wave function in the direction of the vector potential. A symmetrisation was performed to rectify this. Despite the very large magnetic field strength used, the change in the wave function is small. Because of this, we anticipate that the background field approach of determining the magnetic moment of baryons to be effective, even in a strong background field. Full results of this investigation can be found in Ref. [17].

\section{Acknowledgments}

This research was undertaken on the NCI National Facility in Canberra, Australia, which is supported by the Australian Commonwealth Government. We also thank eResearch SA for generous grants of supercomputing time which have enabled this project. This research is supported by the Australian Research Council.

\section{References}

[1] B. Velikson and D. Weingarten, Nucl. Phys. B 249, 433 (1985).

[2] R. Gupta, D. Daniel and J. Grandy, Phys. Rev. D 48 (1993) 3330 [arXiv:hep-lat/9304009].

[3] M. W. Hecht and T. A. DeGrand, Phys. Rev. D 46, 2155 (1992).

[4] J. Smit and J. C. Vink, Nucl. Phys. B 286, 485 (1987).

[5] G. Martinelli, G. Parisi, R. Petronzio and F. Rapuano, Phys. Lett. B 116, 434 (1982).

[6] C. W. Bernard, T. Draper, K. Olynyk and M. Rushton, Phys. Rev. Lett. 49, 1076 (1982).

[7] F. X. Lee, R. Kelly, L. Zhou and W. Wilcox, Phys. Lett. B 627, 71 (2005) [arXiv:hep-lat/0509067].

[8] M. Burkardt, D. B. Leinweber and X. m. Jin, Phys. Lett. B 385 (1996) 52 [arXiv:hep-ph/9604450].

[9] F. X. Lee, L. Zhou, W. Wilcox and J. C. Christensen, Phys. Rev. D 73, 034503 (2006) [arXiv:hep-lat/0509065].

[10] M. Luscher and P. Weisz, Commun. Math. Phys. 97, 59 (1985) [Erratum-ibid. 98, 433 (1985)].

[11] J. M. Zanotti, D. B. Leinweber, W. Melnitchouk, A. G. Williams and J. B. Zhang, Lect. Notes Phys. 663, 199 (2005) [arXiv:hep-lat/0407039].

[12] C. T. H. Davies et al., Phys. Rev. D 37, 1581 (1988).

[13] M. Anselmino, E. Predazzi, S. Ekelin, S. Fredriksson and D. B. Lichtenberg, Rev. Mod. Phys. 65, 1199 (1993).

[14] C. Alexandrou, P. de Forcrand and B. Lucini, PoS LAT2005, 053 (2006) [arXiv:hep-lat/0509113].

[15] C. Alexandrou, Ph. de Forcrand and B. Lucini, Phys. Rev. Lett. 97, 222002 (2006) [arXiv:hep-lat/0609004].

[16] T. DeGrand, Z. Liu and S. Schaefer, Phys. Rev. D 77, 034505 (2008) [arXiv:0712.0254 [hep-ph]].

[17] D. S. Roberts, P. O. Bowman, W. Kamleh and D. B. Leinweber [arXiv:1011.1975 [hep-lat]] 